\title{
Bipartite Graphs Associated with Pell, Mersenne and Perrin Numbers
}

\begin{abstract}
Ahmet Öteleş
Abstract

In this paper, we consider the relationships between the numbers of perfect matchings (1-factors) of bipartite graphs and Pell, Mersenne and Perrin Numbers. Then we give some Maple procedures in order to calculate the numbers of perfect matchings of these bipartite graphs.
\end{abstract}

\section{Introduction}

The well-known integer sequences (e.g., Fibonacci, Pell) provide invaluable opportunities for exploration, and contribute handsomely to the beauty of mathematics, especially number theory $[1,2]$.

The Pell sequence $\{P(n)\}$ is defined by the recurrence relation, for $n \geq 2$

$$
P(n)=2 P(n-1)+P(n-2)
$$

with $P(0)=0$ and $P(1)=1$ [3]. The number $P(n)$ is called $n$th Pell number. The Pell sequence is named as $A 000129$ in [4].

The Mersenne sequence $\{M(n)\}$ is defined by the recurrence relation, for $n \geq 2$

$$
M(n)=2 M(n-1)+1
$$

with $M(0)=0$ and $M(1)=1[5]$. The number $M(n)$ is called $n$th Mersenne number. The Mersenne sequence is named as A000225 in [4].

Key Words: Perfect matching, permanent, Pell number, Mersenne number, Perrin number.

2010 Mathematics Subject Classification: Primary 11B39, 05C50; Secondary 15A15.

Received: 21.05.2018

Accepted: 05.09.2018 
The Perrin sequence $\{R(n)\}$ is defined by the recurrence relation, for $n>2$

$$
R(n)=R(n-2)+R(n-3)
$$

with $R(0)=3, R(1)=0 R(2)=2$. The number $R(n)$ is called $n$th Perrin number [6]. The Perrin sequence is named as $A 001608$ in [4].

The first few values of these sequences can be seen at the following table:

\begin{tabular}{c|cccccccccccc}
$n$ & 0 & 1 & 2 & 3 & 4 & 5 & 6 & 7 & 8 & 9 & 10 & $\ldots$ \\
\hline$P(n)$ & 0 & 1 & 2 & 5 & 12 & 29 & 70 & 169 & 408 & 985 & 2378 & $\ldots$ \\
$M(n)$ & 0 & 1 & 3 & 7 & 15 & 31 & 63 & 127 & 255 & 511 & 1023 & $\ldots$ \\
$R(n)$ & 3 & 0 & 2 & 3 & 2 & 5 & 5 & 7 & 10 & 12 & 17 & $\ldots$
\end{tabular}

The investigation of the properties of bipartite graphs was begun by König. His work was motivated by an attempt to give a new approach to the investigation of matrices on determinants of matrices. As a practical matter, bipartite graphs form a model of the interaction between two different types of objects. For example; social network analysis, railway optimization problem, marriage problem, etc [7]. The enumeration or actual construction of perfect matching of a bipartite graph has many applications, for example, in maximal flow problems and in assignment and scheduling problems arising in operational research [8]. The number of perfect matchings of bipartite graphs also plays a significant role in organic chemistry [9].

A bipartite graph $G$ is a graph whose vertex set $V$ can be partitioned into two subsets $V_{1}$ and $V_{2}$ such that every edge of $G$ joins a vertex in $V_{1}$ and a vertex in $V_{2}$. A perfect matching (or 1 -factor) of a graph is a matching in which each vertex has exactly one edge incident on it. Namely, every vertex in the graph has degree 1. Let $A(G)$ be adjacency matrix of the bipartite graph $G$ and $\mu(G)$ denote the number of perfect matchings of $G$. Then, one can find the following fact in $[8]: \mu(G)=\sqrt{\operatorname{per}(A(G))}$.

Let $G$ be a bipartite graph whose vertex set $V$ is partitioned into two subsets $V_{1}$ and $V_{2}$ such that $\left|V_{1}\right|=\left|V_{2}\right|=n$. We construct the bipartite adjacent matrix $B(G)=\left(b_{i j}\right)$ of $G$ as following: $b_{i j}=1$ if and only if $G$ contains an edge from $v_{i} \in V_{1}$ to $v_{j} \in V_{2}$, and otherwise $b_{i j}=0$. Then, the number of perfect matchings of bipartite graph $G$ is equal to the permanent of its bipartite adjacency matrix [8].

The permanent of an $n \times n$ matrix $A=\left(a_{i j}\right)$ is defined by

$$
\operatorname{per}(A)=\sum_{\sigma \epsilon S_{n}} \prod_{i=1}^{n} a_{i \sigma(i)}
$$

where the summation extends over all permutations $\sigma$ of the symmetric group $S_{n}$. The permanent of a matrix is analogous to the determinant, where all of 
the signs used in the Laplace expansion of minors are positive. One can find the basic properties and more applications of permanents $[8,9,10,11,12,13]$.

Permanents have many applications in physics, chemistry and electrical engineering. Some of the most important applications of permanents are via graph theory. A more difficult problem with many applications is the enumeration of perfect matchings of a graph [8]. Therefore, counting the number of perfect matchings in bipartite graphs has been very popular problem.

One can find so many studies on the relationship between the number of perfect matchings of bipartite graphs and the well-known integer sequences $[14,15,16,17,18,19,20,21,22,23,24,25,26]$.

In this paper, we define three $n \times n(0,1)$-matrices which correspond to the adjacency matrices of some bipartite graphs. Then we show that the numbers of perfect matchings of these bipartite graphs are equal to Pell, Mersenne and Perrin numbers, respectively. Finally, we give some Maple procedures regarding our calculations.

\section{Main Results}

Let $A=\left[a_{i j}\right]$ be an $m \times n$ real matrix with row vectors $\alpha_{1}, \alpha_{2}, \ldots, \alpha_{m}$. We say $A$ is contractible on column (resp. row) $k$ if column (resp. row) $k$ contains exactly two nonzero entries. Suppose $A$ is contractible on column $k$ with $a_{i k} \neq 0 \neq a_{j k}$ and $i \neq j$. Then the $(m-1) \times(n-1)$ matrix $A_{i j: k}$ obtained from $A$ by replacing row $i$ with $a_{j k} \alpha_{i}+a_{i k} \alpha_{j}$ and deleting row $j$ and column $k$ is called the contraction of $A$ on column $k$ relative to rows $i$ and $j$. If $A$ is contractible on row $k$ with $a_{k i} \neq 0 \neq a_{k j}$ and $i \neq j$, then the matrix $A_{k: i j}=\left[A_{i j: k}^{T}\right]^{T}$ is called the contraction of $A$ on row $k$ relative to columns $i$ and $j$. We say that $A$ can be contracted to a matrix $B$ if either $B=A$ or there exist matrices $A_{0}, A_{1}, \ldots, A_{t}(t \geq 1)$ such that $A_{0}=A, A_{t}=B$, and $A_{r}$ is a contraction of $A_{r-1}$ for $r=1, \ldots, t[10]$.

Brualdi and Gibson [10] proved the following result about the permanent of a matrix.

Lemma 2.1. Let $A$ be a nonnegative integral matrix of order $n$ for $n>1$ and let $B$ be a contraction of $A$. Then

$$
\operatorname{per} A=\operatorname{per} B
$$


Let $H_{n}$ be an $n \times n(0,1)$-matrix having form

$$
H_{n}=\left(\begin{array}{cccccccc}
1 & 1 & 0 & 0 & \cdots & \cdots & \cdots & 0 \\
1 & 1 & 0 & 1 & 0 & & & \vdots \\
0 & 1 & 1 & 1 & \ddots & \ddots & & \vdots \\
\vdots & 0 & 1 & \ddots & \ddots & \frac{1+(-1)^{j}}{2} & \ddots & \vdots \\
\vdots & & \ddots & \ddots & \ddots & \frac{1+(-1)^{j}}{2} & \ddots & 0 \\
\vdots & & & \ddots & \ddots & \ddots & \ddots & \frac{1+(-1)^{n}}{2} \\
\vdots & & & & 0 & 1 & 1 & \frac{1+(-1)^{n}}{2} \\
0 & \cdots & \ldots & \cdots & \cdots & 0 & 1 & 1
\end{array}\right)
$$

where

$$
h_{i j}= \begin{cases}1, & \text { if } j-i=-1 \text { or } j-i=0, \\ \frac{1+(-1)^{j}}{2}, & \text { if } j-i=1 \text { or } j-i=2, \\ 0, & \text { otherwise. }\end{cases}
$$

Theorem 2.2. Let $G\left(H_{n}\right)$ be the bipartite graph with bipartite adjacency matrix $H_{n}$ given by (4). Then, the number of perfect matchings of $G\left(H_{n}\right)$ is $\left\lfloor\frac{n+2}{2}\right\rfloor$ th Pell number $P\left(\left\lfloor\frac{n+2}{2}\right\rfloor\right)$, where $\lfloor x\rfloor$ is the largest integer less than or equal to $x$.

Proof. Let $H_{n}^{r}$ be the $r$ th contraction of the matrix $H_{n}, 1 \leq r \leq n-2$. By definition of $H_{n}$, the matrix $H_{n}$ can be contracted on column 1 so that

$$
H_{n}^{1}=\left(\begin{array}{cccccccc}
2 & 0 & 1 & 0 & \cdots & \cdots & \cdots & 0 \\
1 & 1 & 1 & 0 & 0 & & & \vdots \\
0 & 1 & 1 & 0 & \ddots & \ddots & & \vdots \\
\vdots & 0 & 1 & \ddots & \ddots & \frac{1+(-1)^{j}}{2} & \ddots & \vdots \\
\vdots & & \ddots & \ddots & \ddots & \frac{1+(-1)^{j}}{2} & \ddots & 0 \\
\vdots & & & \ddots & \ddots & \ddots & \ddots & \frac{1+(-1)^{n}}{2} \\
\vdots & & & & 0 & 1 & 1 & \frac{1+(-1)^{n}}{2} \\
0 & \ldots & \ldots & \ldots & \ldots & 0 & 1 & 1
\end{array}\right) .
$$


Since the matrix $H_{n}^{1}$ can be contracted on column 1 and $P(2)=2, P(1)=1$

$$
\begin{aligned}
& H_{n}^{2}=\left(\begin{array}{cccccccc}
2 & 3 & 0 & 0 & \cdots & \cdots & \cdots & 0 \\
1 & 1 & 0 & 1 & 0 & & & \vdots \\
0 & 1 & 1 & 1 & \ddots & \ddots & & \vdots \\
\vdots & 0 & 1 & \ddots & \ddots & \frac{1+(-1)^{j}}{2} & \ddots & \vdots \\
\vdots & & \ddots & \ddots & \ddots & \frac{1+(-1)^{j}}{2} & \ddots & 0 \\
\vdots & & & \ddots & \ddots & \ddots & \ddots & \frac{1+(-1)^{n}}{2} \\
\vdots & & & & 0 & 1 & 1 & \frac{1+(-1)^{n}}{2} \\
0 & \cdots & \ldots & \cdots & \cdots & 0 & 1 & 1
\end{array}\right)
\end{aligned}
$$

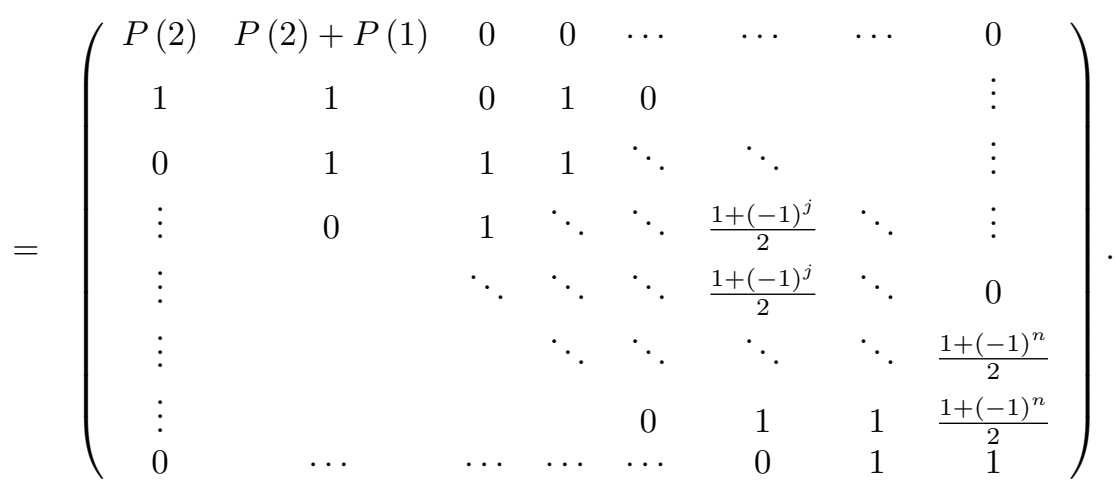

Furthermore, the matrix $H_{n}^{2}$ can be contracted on column 1 and taking into account (1), so that

$$
H_{n}^{3}=\left(\begin{array}{cccccccc}
P(3) & 0 & P(2) & 0 & \cdots & \cdots & \cdots & 0 \\
1 & 1 & 1 & 0 & 0 & & & \vdots \\
0 & 1 & 1 & 0 & \ddots & \ddots & & \vdots \\
\vdots & 0 & 1 & \ddots & \ddots & \frac{1+(-1)^{j}}{2} & \ddots & \vdots \\
\vdots & & \ddots & \ddots & \ddots & \frac{1+(-1)^{j}}{2} & \ddots & 0 \\
\vdots & & & \ddots & \ddots & \ddots & \ddots & \frac{1+(-1)^{n}}{2} \\
\vdots & & & & 0 & 1 & 1 & \frac{1+(-1)^{n}}{2} \\
0 & \ldots & \ldots & \ldots & \cdots & 0 & 1 & 1
\end{array}\right) .
$$


Continuing this process, we derive the $r$ th contraction of $H_{n}$ as: If $r$ is odd,

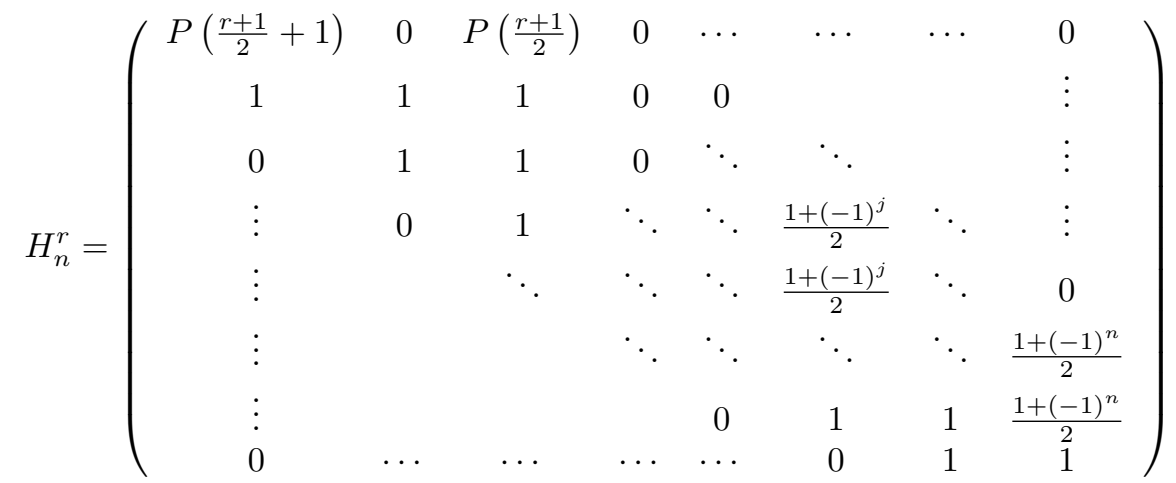

and if $r$ is even,

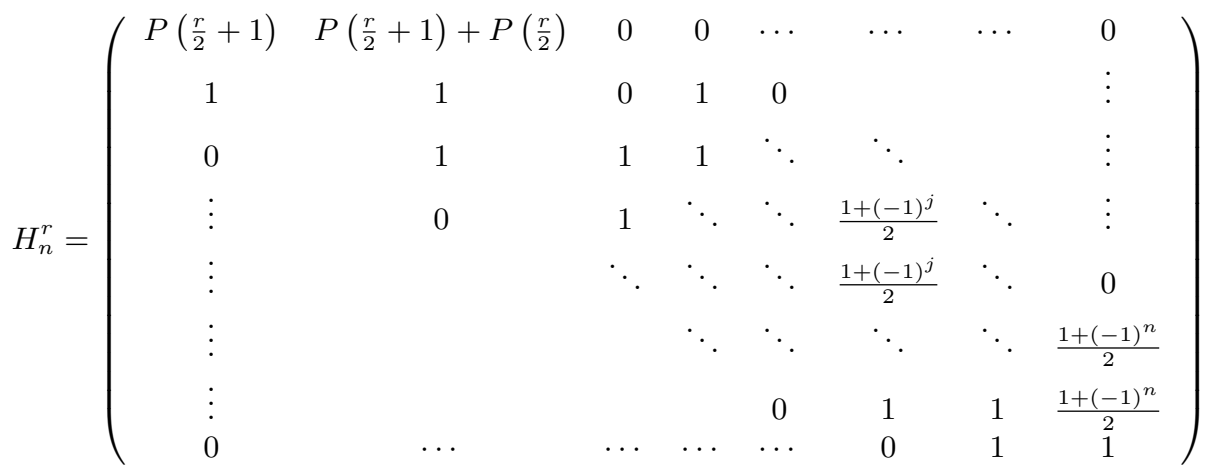

for $3 \leq r \leq n-3$. Notice that if $n$ is odd (even) then $r=n-3$ is even (odd). Consequently,

$$
H_{n}^{n-3}=\left\{\begin{array}{ccc}
P\left(\frac{n-1}{2}\right) & P\left(\frac{n-1}{2}\right)+P\left(\frac{n-1}{2}-1\right) & 0 \\
1 & 1 & 0 \\
0 & 1 & 1
\end{array}\right) \text { if } n \text { is odd }
$$

which, by contraction of $H_{n}^{n-3}$ on column 1 and taking into account (1), gives

$$
H_{n}^{n-2}=\left\{\begin{array}{cc}
\left(\begin{array}{cc}
P\left(\frac{n+1}{2}\right) & 0 \\
1 & 1
\end{array}\right), & \text { if } n \text { is odd } \\
P\left(\frac{n}{2}\right) & P\left(\frac{n}{2}\right)+P\left(\frac{n}{2}-1\right) \\
1 & 1
\end{array}\right), \quad \text { if } n \text { is even. }
$$


By applying the equation (3) to the expression (5) and taking into account (1), we obtain

$$
\text { per } H_{n}=\operatorname{per} H_{n}^{n-2}= \begin{cases}P\left(\frac{n+1}{2}\right), & \text { if } n \text { is odd, } \\ P\left(\frac{n+2}{2}\right), & \text { if } n \text { is even, }\end{cases}
$$

which is deduced that per $H_{n}=P\left(\left\lfloor\frac{n+2}{2}\right\rfloor\right)$. So, the proof is completed.

Let $K_{n}$ be an $n \times n(0,1)$-matrix having form

$$
K_{n}=\left(\begin{array}{cccccccc}
1 & 0 & 1 & 0 & \ldots & \frac{1-(-1)^{j}}{2} & \ldots & \frac{1-(-1)^{n}}{2} \\
1 & 1 & 1 & 0 & \ldots & \ldots & \ldots & 0 \\
0 & 1 & 1 & 0 & 0 & & & \vdots \\
\vdots & \ddots & \ddots & \ddots & \ddots & \ddots & & \vdots \\
\vdots & & \ddots & 1 & 1 & \frac{1-(-1)^{j}}{2} & \ddots & \vdots \\
\vdots & & & \ddots & \ddots & \ddots & \ddots & 0 \\
\vdots & & & & \ddots & \ddots & 1 & \frac{1-(-1)^{n}}{2} \\
0 & \ldots & \ldots & \ldots & \ldots & 0 & 1 & 1
\end{array}\right)
$$

where

$$
k_{i j}= \begin{cases}1, & \text { if } j-i=-1 \text { or } j-i=0, \\ \frac{1-(-1)^{j}}{2}, & \text { if } i=1 \text { or } j-i=1, \\ 0, & \text { otherwise. }\end{cases}
$$

Theorem 2.3. Let $G\left(K_{n}\right)$ be the bipartite graph with bipartite adjacency matrix $K_{n}$ given by (6). Then, the number of perfect matchings of $G\left(K_{n}\right)$ is $\left\lfloor\frac{n+1}{2}\right\rfloor$ th Mersenne number $M\left(\left\lfloor\frac{n+1}{2}\right\rfloor\right)$, where $\lfloor x\rfloor$ is the largest integer less than or equal to $x$.

Proof. Let $K_{n}^{r}$ be the $r$ th contraction of $B_{n}$ for $1 \leq r \leq n-3$. By applying successive contractions to the matrices $K_{n}^{r}$ for $1 \leq r \leq n-3$ according to their first columns, we get

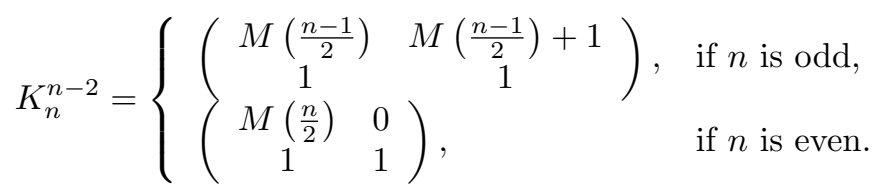

By applying the equation (3) to the expression (7) and taking into account (2), we obtain

$$
\operatorname{per} K_{n}=\operatorname{per} K_{n}^{n-2}= \begin{cases}M\left(\frac{n+1}{2}\right), & \text { if } n \text { is odd, } \\ M\left(\frac{n}{2}\right), & \text { if } n \text { is even, }\end{cases}
$$


which is deduced that $\operatorname{per} K_{n}=M\left(\left\lfloor\frac{n+1}{2}\right\rfloor\right)$. So, it is desired.

In [23, Theorem 2], we can reach the following result regarding the relationship between Perrin numbers and the permanent of a certain upper Hessenberg matrix.

Theorem 2.4. Let $B_{n}=\left(b_{i j}\right)$ be the $n \times n$ matrix such that $b_{i j}=2$ if and only if $i=1$ and $j=1, b_{i j}=3$ if and only if $i=1$ and $j=2, b_{i j}=1$ if and only if $j-i=-1$ or $i>1$ and $j-i=1$, or $i>1$ and $j-i=2$ and otherwise 0. Clearly,

$$
B_{n}=\left(\begin{array}{ccccccc}
2 & 3 & 0 & 0 & \cdots & \cdots & 0 \\
1 & 0 & 1 & 1 & 0 & & \vdots \\
0 & 1 & 0 & 1 & 1 & \ddots & \vdots \\
\vdots & 0 & 1 & 0 & \ddots & \ddots & 0 \\
\vdots & & \ddots & \ddots & \ddots & 1 & 1 \\
\vdots & & & \ddots & 1 & 0 & 1 \\
0 & \cdots & \cdots & \cdots & 0 & 1 & 0
\end{array}\right) .
$$

Then the permanent of $B_{n}$ is the $(n+1)$ st Perrin number $R(n+1)$.

Let $S_{n}=\left(s_{i j}\right)$ be the $n \times n(0,1)$-matrix defined by $s_{i j}=1$ if and only if $|j-i|=1$ or $j-i=2$. Let $T_{n}=\left(t_{i j}\right)$ be the $n \times n$ tridiagonal $(0,1)$-matrix with $t_{11}=t_{22}=1$. Let $U_{n}=\left(u_{i j}\right)$ be the $n \times n \quad(0,1)$-matrix with $u_{35}=1$. Then we can give the following theorem.

Theorem 2.5. Let $G\left(L_{n}\right)$ be the bipartite graph with bipartite adjacency matrix $L_{n}=S_{n}+T_{n}+U_{n}$ for $n \geq 3$. Then, the number of perfect matchings of $G\left(L_{n}\right)$ is $(n-1)$ st Perrin number $R(n-1)$.

Proof. Let $L_{n}^{r}$ be the $r$ th contraction of the matrix $L_{n}, 1 \leq r \leq n-2$. By definition of $L_{n}$, the matrix $L_{n}$ can be contracted on column 1 so that

$$
L_{n}^{1}=\left(\begin{array}{cccccccc}
2 & 2 & 1 & 0 & \cdots & \cdots & \cdots & 0 \\
1 & 0 & 1 & 0 & 0 & & & \vdots \\
0 & 1 & 0 & 1 & 1 & 0 & & \vdots \\
\vdots & 0 & 1 & 0 & 1 & 1 & \ddots & \vdots \\
\vdots & & \ddots & \ddots & \ddots & \ddots & \ddots & 0 \\
\vdots & & & \ddots & \ddots & 0 & 1 & 1 \\
\vdots & & & & 0 & 1 & 0 & 1 \\
0 & \cdots & \cdots & \cdots & \cdots & 0 & 1 & 0
\end{array}\right) .
$$


If the matrix $L_{n}^{1}$ can be contracted on column 1 , then

$$
L_{n}^{2}=\left(\begin{array}{cccccccc}
2 & 3 & 0 & 0 & \cdots & \cdots & \cdots & 0 \\
1 & 0 & 1 & 1 & 0 & & & \vdots \\
0 & 1 & 0 & 1 & 1 & 0 & & \vdots \\
\vdots & 0 & 1 & 0 & 1 & 1 & \ddots & \vdots \\
\vdots & & \ddots & \ddots & \ddots & \ddots & \ddots & 0 \\
\vdots & & & \ddots & \ddots & 0 & 1 & 1 \\
\vdots & & & & 0 & 1 & 0 & 1 \\
0 & \cdots & \cdots & \cdots & \cdots & 0 & 1 & 0
\end{array}\right)
$$

which is equal to $B_{n-2}$, where $B_{n}$ is the matrix defined by (8). By applying the equation (3) to the expression (9) and taking into account Theorem 2.4, we obtain

$$
\operatorname{per} L_{n}=\operatorname{per} L_{n}^{2}=\operatorname{per} B_{n-2}=R(n-1),
$$

which is desired.

Appendix A. The following Maple procedure calculates the numbers of perfect matchings of bipartite graph $G\left(H_{n}\right)$ given in Theorem 2.2.

restart:

with(LinearAlgebra):

permanent: $=\operatorname{proc}(\mathrm{n})$

local i,j,r,h,H;

$\mathrm{h}:=(\mathrm{i}, \mathrm{j})->\operatorname{piecewise}\left(\mathrm{j}-\mathrm{i}=-1,1, \mathrm{j}-\mathrm{i}=0,1, \mathrm{j}-\mathrm{i}=1,\left(1+(-1)^{j}\right) / 2, j-i=2,(1+\right.$ $\left.\left.(-1)^{j}\right) / 2,0\right)$;

$\mathrm{H}:=\operatorname{Matrix}(\mathrm{n}, \mathrm{n}, \mathrm{h})$ :

for $r$ from 0 to $n-2$ do

$\operatorname{print}(\mathrm{r}, \mathrm{H})$ :

for $\mathrm{j}$ from 2 to $\mathrm{n}-\mathrm{r}$ do

$\mathrm{H}[1, \mathrm{j}]:=\mathrm{H}[2,1] * \mathrm{H}[1, \mathrm{j}]+\mathrm{H}[1,1] * \mathrm{H}[2, \mathrm{j}]$ :

od:

$\mathrm{H}:=\operatorname{DeleteRow}(\operatorname{DeleteColumn}(\operatorname{Matrix}(\mathrm{n}-\mathrm{r}, \mathrm{n}-\mathrm{r}, \mathrm{H}), 1), 2)$ :

od:

$\operatorname{print}(\mathrm{r}, \operatorname{eval}(\mathrm{H}))$ :

end proc:with(LinearAlgebra):

permanent(n);

Appendix B. The following Maple procedure calculates the numbers of perfect matchings of bipartite graph $G\left(K_{n}\right)$ given in Theorem 2.3. 
restart:

with(LinearAlgebra):

permanent: $=\operatorname{proc}(\mathrm{n})$

local i,j,r,k,K;

$\mathrm{k}:=(\mathrm{i}, \mathrm{j})->$ piecewise $\left(\mathrm{i}=1,\left(1-(-1)^{j}\right) / 2, j-i=-1,1, j-i=0,1, j-i=\right.$ $\left.1,\left(1-(-1)^{j}\right) / 2,0\right)$

$\mathrm{K}:=\operatorname{Matrix}(\mathrm{n}, \mathrm{n}, \mathrm{k})$ :

for $\mathrm{r}$ from 0 to $\mathrm{n}-2$ do

$\operatorname{print}(\mathrm{r}, \mathrm{K})$ :

for $\mathrm{j}$ from 2 to $\mathrm{n}-\mathrm{r}$ do

$\mathrm{K}[1, \mathrm{j}]:=\mathrm{K}[2,1] * \mathrm{~K}[1, \mathrm{j}]+\mathrm{K}[1,1] * \mathrm{~K}[2, \mathrm{j}]$ :

od:

$\mathrm{K}:=$ DeleteRow $($ DeleteColumn(Matrix(n-r,n-r,K),1),2):

od:

print(r,eval(K)):

end proc:with(LinearAlgebra):

permanent(n);

Appendix C. The following Maple procedure calculates the numbers of perfect matchings of bipartite graph $G\left(L_{n}\right)$ given in Theorem 2.5.

restart:

with(LinearAlgebra):

permanent: $=\operatorname{proc}(\mathrm{n})$

local i,j,r,s,t,u,S,T,U,L;

$\mathrm{s}:=(\mathrm{i}, \mathrm{j})->$ piecewise $(\operatorname{abs}(\mathrm{j}-\mathrm{i})=1,1, \mathrm{j}-\mathrm{i}=2,1,0)$;

$\mathrm{t}:=(\mathrm{i}, \mathrm{j})->$ piecewise $(\mathrm{i}=1$ and $\mathrm{j}=1,1, \mathrm{i}=2$ and $\mathrm{j}=2,1,0)$;

$\mathrm{u}:=(\mathrm{i}, \mathrm{j})->$ piecewise $(\mathrm{i}=3$ and $\mathrm{j}=5,1,0)$;

$\mathrm{S}:=\operatorname{Matrix}(\mathrm{n}, \mathrm{n}, \mathrm{s})$ :

$\mathrm{T}:=\operatorname{Matrix}(\mathrm{n}, \mathrm{n}, \mathrm{t})$ :

$\mathrm{U}:=\operatorname{Matrix}(\mathrm{n}, \mathrm{n}, \mathrm{u})$ :

$\mathrm{L}:=\mathrm{S}+\mathrm{T}-\mathrm{U}$ :

for $\mathrm{r}$ from 0 to $\mathrm{n}-2$ do

$\operatorname{print}(\mathrm{r}, \mathrm{L})$ :

for $\mathrm{j}$ from 2 to $\mathrm{n}-\mathrm{r}$ do

$\mathrm{L}[1, \mathrm{j}]:=\mathrm{L}[2,1]^{*} \mathrm{~L}[1, \mathrm{j}]+\mathrm{L}[1,1]^{*} \mathrm{~L}[2, \mathrm{j}]:$

od:

$\mathrm{L}:=$ DeleteRow $($ DeleteColumn $($ Matrix $(\mathrm{n}-\mathrm{r}, \mathrm{n}-\mathrm{r}, \mathrm{L}), 1), 2)$ :

od:

print(r,eval(L)):

end proc:with(LinearAlgebra):

permanent(n); 
BIPARTITE GRAPH ASSOCIATED WITH PELL, MERSENNE AND PERRIN NUMBERS 119

\section{References}

[1] T. Koshy, Fibonacci and Lucas Numbers with Applications, WileyInterscience, New York, 2001.

[2] T. Koshy, Fibonacci, Lucas, and Pell numbers, and Pascal's triangle, Math. Spectrum, 43(3) (2011), 125-132.

[3] A.F. Horadam, Jacobstal and Pell Curves, Fibonacci Quart., 26 (1988), 79-83.

[4] The OEIS Foundation Inc., The On-Line Encyclopedia of Integer Sequences, https://oeis.or, 2013.

[5] P. Catarino, H. Campos, P. Vasco, On the Mersenne sequence, Annales Mathematicae et Informaticae, 46 (2016), 37-53.

[6] W. Adams, D. Shanks, Strong primality tests that are not sufficient, Mathematics of Computation, 39(159) (1982), 255-300.

[7] A.S. Asratian, T.M.J. Denley, R. Häggkvist, Bipartite Graphs and their Applications, Cambridge Tracts in Mathematics, 131, Cambridge University Press, 1998.

[8] H. Minc, Permanents, Encyclopedia of mathematics and its applications, Addison-Wesley, New York, 1978.

[9] G.W. Wheland, The Theory of Resonant and its Application to Organic Chemistry, Wiley, New York, 1953.

[10] R.A. Brualdi, P.M. Gibson, Convex polyhedra of doubly stochastic matrices I: applications of the permanent function, J. Combin. Theory A, 22 (1977), 194-230.

[11] R.A. Brualdi, D. Cvetkovic, A Combinatorial Approach to Matrix Theory and Its Applications, CRC Press, 2009.

[12] F. Harary, Determinants, permanents and bipartite graphs, Math. Mag., 42 (1969), 146-148.

[13] M. Marcus, H. Minc, Permanents, Amer. Math. Monthly, 72 (1965), 577591.

[14] G.Y. Lee, S.G. Lee, H. G. Shin, On the k-generalized Fibonacci matrix $Q_{k}$, Lin. Alg. Appl., 251 (1997), 73-88. 
[15] G.Y. Lee, k-Lucas numbers and associated bipartite graphs, Lin. Alg. Appl., 320 (2000), 51-61.

[16] W.C. Shiu, Peter C.B. Lam, More on the generalized Fibonacci numbers and associated bipartite graphs, Int. Math. J., 3 (2003), 5-9.

[17] E. Kılıç, D. Tasçı, On families of bipartite graphs associated with sums of Fibonacci and Lucas numbers, Ars Combin., 89 (2008), 31-40.

[18] G.Y. Lee, S.G. Lee, A note on generalized Fibonacci numbers, Fibonacci Quart., 33 (1995), 273-278.

[19] E. Kılıç, D. Taşçı, On the permanents of some tridiagonal matrices with applications to the Fibonacci and Lucas numbers, Rocky Mt. J. Math., 37(6) (2007), 1953-1969.

[20] M. Akbulak, A. Öteleş, On the number of 1-factors of bipartite graphs, Math. Sci. Lett., 2(3) (2013), 1-7.

[21] A. Öteles, On the Number of Perfect Matchings for Some Certain Types of Bipartite Graphs, Filomat, 31(15) (2017), 48094818.

[22] F. Yilmaz and D. Bozkurt, Some properties of Padovan sequence by matrix methods, Ars Combin., 104 (2012), 149-160.

[23] F. Yilmaz and D. Bozkurt, Hessenberg matrices and the Pell and Perrin numbers, J. Number Theory, 131 (2011), 1390-1396.

[24] C.M. da Fonseca, T. Sogabe and F. Yilmaz, Lower k-Hessenberg Matrices and $k$-Fibonacci, Fibonacci-p and Pell $(p, i)$ Number, Gen. Math. Notes, 31(1) (2015), 10-17.

[25] E. Kılıç, D. Tasçı, On families of bipartite graphs associated with sums of generalized order- $k$ Fibonacci and Lucas numbers, Ars Combin., 94 (2008), 13-23.

[26] E. Kılıç, A.P. Stakhov, On the Fibonacci and Lucas p-numbers, their sums, families of bipartite graphs and permanents of certain matrices, Chaos Solitons Fractals, 40(22) (2009), 10-21.

\footnotetext{
Ahmet Öteleş,

Department of Mathematics,

Faculty of Education,

Dicle University,

21280 Diyarbakir, Turkey.

Email: aoteles85@gmail.com
} 